Research Article

\title{
Comparative mitogenomic analyses of Amazona parrots and Psittaciformes
}

Nicholas Costa Barroso Lima ${ }^{1,2,8}$, André Elias Rodrigues Soares ${ }^{2}$, Luiz Gonzaga de Paula Almeida ${ }^{2}$, Igor Rodrigues da $\operatorname{Costa}^{1}$, Fernanda Midori Sato ${ }^{4}$, Patricia Schneider ${ }^{3}$, Alexandre Aleixo ${ }^{5}$, Maria Paula Schneider ${ }^{3}$, Fabrício R. Santos ${ }^{6}$, Claudio V. Mello ${ }^{7}$, Cristina Miyaki ${ }^{4}$, Ana Tereza R. Vasconcelos ${ }^{2}$ and Francisco Prosdocimi ${ }^{1}$ iD

${ }^{1}$ Laboratório de Genômica e Biodiversidade, Instituto de Bioquímica Médica Leopoldo de Meis, Universidade Federal do Rio de Janeiro, Rio de Janeiro, RJ, Brazil.

${ }^{2}$ Laboratório de Bioinformática, Laboratório Nacional de Computação Científica, Petrópolis, RJ, Brazil.

${ }^{3}$ Departamento de Genética, Centro de Ciências Biológicas, Universidade Federal do Pará, Belém, PA,

Brazil.

${ }^{4}$ Laboratório de Genética e Evolução Molecular de Aves, Departamento de Genética e Biologia Evolutiva, Instituto de Biociências, Universidade de São Paulo, SP, Brazil.

${ }^{5}$ Coordenação de Zoologia, Museu Paraense Emilio Goeldi, Belém, PA, Brazil.

${ }^{6}$ Departamento de Biologia Geral, Instituto de Ciências Biológicas, Universidade Federal de Minas Gerais, Belo Horizonte, MG, Brazil.

${ }^{7}$ Department of Behavioral Neuroscience, Oregon Health \& Science University, Portland, OR, USA.

${ }^{8}$ Departamento de Bioquímica e Biologia Molecular, Universidade Federal do Ceará, Fortaleza, CE, Brazil.

\begin{abstract}
Amazon parrots are long-lived birds with highly developed cognitive skills, including vocal learning. Several parrot mitogenomes have been sequenced, but important aspects of their organization and evolution are not fully understood or have limited experimental support. The main aim of the present study was to describe the mitogenome of the blue-fronted Amazon, Amazona aestiva, and compare it to other mitogenomes from the genus Amazona and the order Psittaciformes. We observed that mitogenomes are highly conserved among Amazon parrots, and a detailed analysis of their duplicated control regions revealed conserved blocks. Population level analyses indicated that the specimen analyzed here seems to be close to $A$. aestiva individuals from Bahia state. Evolutionary relationships of 41 Psittaciformes species and three outgroups were inferred by BEAST. All relationships were retrieved with high support.
\end{abstract}

Keywords: Next-generation-sequencing, Psittacidae, mitogenomics, control region.

Received: February 06, 2017; Accepted: January 22, 2018.

\section{Introduction}

Vertebrate mitochondrial genomes (mitogenomes) consist of a circular DNA molecule of about $16 \mathrm{~Kb}$ that contains 37 intronless genes. This tightly packed organization with little overlap of genomic features is thought to be the result of selective pressure (Mindell, 1999; Boore, 1999; Ingman and Gyllensten, 2009). The chicken mitogenome was the first avian one to be completely sequenced and annotated (Desjardins and Morais, 1990) and revealed a remarkable difference in gene order in comparison to

Send correspondence to Francisco Prosdocimi. Laboratório de Genômica e Biodiversidade, Instituto de Bioquímica Médica Leopoldo de Meis, Universidade Federal do Rio de Janeiro, Centro de Ciências da Saúde (CCS) Bloco E Sala 022, Cidade UniversitáriaIlha do Fundão, 21.941-902 Rio de Janeiro, RJ, Brazil. E-mail: prosdocimi@bioqmed.ufrj.br. other vertebrate mitogenomes. Its gene arrangement was initially named as "typical" or "ancestral" avian gene order (Desjardins and Morais, 1990). Since then, hundreds of other avian mitochondrial genomes have been described. In October, 2017, 635 complete mitogenomes were available for Aves in GenBank.

Psittaciformes is one of the most conspicuous avian orders, with species characterized by high longevity (Munshi-South et al., 2006; Young et al., 2012) and advanced cognitive abilities (Pepperberg, 1990; Pepperberg and Funk, 1990; Borsari and Ottoni, 2005), including vocal learning (Farabaugh, 1996; Brauth et al., 1997; Pepperberg, 2002, 2010; Plummer and Striedter, 2002). Regarding the mitogenome, many clades of the family Psittacidae present duplicate copies of the control region (CR) as a re- 
sult of at least six independent duplication events (Schirtzinger et al., 2012). The Amazon parrots, genus Amazona, are among the psittacids that have duplicated CRs, as initially shown by Eberhard et al. (2001). The duplication event that gave rise to a duplicated CR in these parrots also resulted in two pseudogenes, pseudo-ND6 and pseudotRNA-Glu at the 5'-end of CR1 (Eberhard and Wright, 2016). Mitogenomes have been fully sequenced for only two Amazona species, the yellow-shouldered parrot, Amazona barbadensis (Urantowka et al., 2013) and the yellow-crowned amazon, Amazona ochrocephala (Eberhard et al., 2001).

In order to thoroughly describe and compare mitogenomes of Amazon parrots, we sequenced, assembled and annotated for the first time the complete mitogenome of the blue-fronted parrot (also known as turquoise-fronted parrot or blue-fronted amazon), Amazona aestiva. This effort was conducted in the context of the whole genome sequencing project that is under way for this species. We also compared the two CRs of A. aestiva with all CRs available for Amazon parrots and identified conserved domains, sequence motifs, and substitution patterns by comparative genomics approaches using both bioinformatics algorithms and manual annotation. Furthermore, all 40 mitogenomes of Psittaciformes species available (as of October, 2017) were used in phylogenomic analyses. Finally, as previous genetic studies suggest that $A$. aestiva and $A$. ochrocephala are not reciprocally monophyletic (Eberhard et al., 2004; Ribas et al., 2007; Caparroz et al., 2009; Chaves et al., 2014), we compared the sequence of the COI gene from the mitogenome described here with those of other individuals of A. aestiva and A. ochrocephala from various localities in South America to confirm if this individual has a typical $A$. aestiva sequence.

\section{Materials and Methods}

\section{Sample collection and DNA sequencing}

A blood sample was previously (2013) obtained by venipuncture from a captive born male Amazona aestiva according to a procedure approved by the Animal Ethics Committee of the Universidade Federal de Minas Gerais (UFMG, 202/2007). This individual (FVVF132) is still alive and legally owned by a private breeder, and its blood sample (B04212) is deposited at UFMG's Centro de Coleções Taxonômicas in Brazil. Total DNA was extracted using DNeasy Blood \& Tissue Kit (Qiagen). Three libraries with insert sizes of $200 \mathrm{bp}, 3 \mathrm{Kbp}$, and $5 \mathrm{Kbp}$ (Table 1) were sequenced in an Illumina HiSeq for the assembly of the nuclear genome. We mapped all the resulting reads on the mitogenome of Amazona barbadensis (GenBank accession number JX524615.1) using Newbler (v 2.9) in order to select putative mitochondrial DNA reads.
Table 1 - Number of reads per A. aestiva sequencing library. Total number of raw reads per library, reads that were mapped to the Amazona barbadensis mitogenome, and total number of reads used in the assembly of the mitogenome $A$. aestiva.

\begin{tabular}{lccc}
\hline Library & Raw reads & Mapped reads & Assembled reads \\
\hline $200 \mathrm{bp}$ & $398,656,732$ & 25,638 & 15,194 \\
$3 \mathrm{Kbp}$ & $160,000,000$ & 3,310 & 844 \\
$5 \mathrm{Kbp}$ & $417,221,300$ & 13,320 & 2,967 \\
Total & $975,878,032$ & 42,268 & 19,005 \\
\hline
\end{tabular}

\section{Assembly and annotation of the blue-fronted parrot mitochondrial genome}

Reads that mapped against the $A$. barbadensis mitogenome were selected and assembled using MASURCA (v 2.3.2) (Zimin et al., 2013) to produce a circular mitogenome. A total of 19,005 reads were assembled (Table 1) and sequence annotation was performed using MITOS Web Server (Bernt et al., 2013). Artemis Genome Browser (Rutherford et al., 2000) was used for manual annotation of mitogenomic features. We ran tRNAscan-SE (Lowe and Eddy, 1997) to confirm tRNA annotation. BLAST searches of amino acid sequences were performed to check for precise gene boundaries. Tablet (Milne et al., 2013) was used to verify the sequence coverage along the assembled mitogenome. Conserved regions within the control region - CR - (ETAS, boxes and conserved sequence blocks) were defined by manual annotation based on an alignment of the CR from five taxa of the genus Amazona.

\section{Identification of control region domains and conserved boxes}

After manual annotation of the CRs from four Amazona taxa (A. aestiva, A. barbadensis, A. farinosa, A. ochrocephala auropalliata and $A$. ochrocephala oratrix), we defined the precise boundaries of the domains as follows: the limit between domains I and II was defined using a 45 nucleotide window graph (Ruokonen and Kvist, 2002; $\mathrm{ADN}$ riche en software), where the number of adenines dropped to a minimum and the number of guanines started to increase (Figure S1). We also used the beginning of the F-box, which marks the start of a long stretch of conserved sequence in the alignment, for determining this boundary. To define the limit between domains II and III we followed Ruokonen and Kvist (2002) and considered the start of block CSB-1 as the boundary. Therefore, domain II was placed upstream of CSB-1 and domain III was defined as downstream of its first base. The identification of CSBs and conserved boxes was performed by manual inspection of alignments of these elements to the two copies of the CR in $A$. aestiva and other species, in agreement with previous definitions (Eberhard and Wright, 2016). 


\section{Whole mitogenome alignment of Amazon parrots}

The $A$. aestiva mitogenome was aligned to the mitogenomes of $A$. barbadensis and A. ochrocephala (GenBank accession number NC_027840.1). NUCmer was used for the alignment, and delta-filter and show-snps, from the same program package (Kurtz et al., 2004), were used to mark nucleotide differences in the pairwise comparisons between the mitogenomes of $A$. aestiva and the other two species. The location and type of mismatches were tabulated and verified relative to the genomic features of the $A$. aestiva mitogenome (Table 2 ).

\section{Psittaciformes mitochondrial phylogenomics}

We aligned all the complete mitochondrial genomes from 40 species of the order Psittaciformes from GenBank (October, 2017; Table S1), our A. aestiva mitochondrion plus three avian outgroups (chicken, zebra finch and peregrine falcon) using MUSCLE (Edgar, 2004), and visually inspected the alignment in SeaView v4.5.4 (Gouy et al., 2010). We partitioned the alignment in order to accommodate for the variable evolutionary rates along the different regions of the mitochondrion. For our phylogenetic analyses we used all coding genes as well as the $12 \mathrm{~S}$ and $16 \mathrm{~S}$ ribosomal RNA genes, which accounts for the majority of the mitochondrial genomes. We split the dataset into four partitions: $12 \mathrm{~S}$ and $16 \mathrm{~S}$ ribosomal genes in one partition, and three partitions for the concatenated coding genes, according to their codon position. We used PartitionFinder (Lanfear et al., 2017) to select the evolutionary model for each partition. We estimated the Psittaciformes phylogenetic relationships using BEAST v1.8.4(Drummond et al., 2012). We assumed a lognormal uncorrelated relaxed clock (Drummond et al., 2006) for each partition, and a GTR $+\mathrm{G}$ (4 categories) nucleotide substitution model, with a BirthDeath speciation process (Gernhard, 2008) for the tree prior. We calibrated the molecular clock by placing three distinct normal priors on the age of divergence between: 1) Neoaves and Galloanseres, 87 Mya \pm 10 Mya; 2) Falconiformes and Passerimorpha, 60 Mya \pm 5 Mya; and 3) Psittaciformes and Passeriformes, 50 Mya \pm 5 Mya (Jarvis et al., 2014; Prum et al., 2015). We ran three distinct MCMC chains for 50 million states, discarding the first $10 \%$ as burn-in. We inspected for convergence using Tracer v1.6, and built a maximum clade credibility tree using TreeAnnotator v1.8 (Drummond et al., 2012).

\section{Haplotype network}

Previous molecular phylogenies have shown that $A$. aestiva and $A$. ochrocephala are not reciprocally monophyletic (Eberhard et al., 2004; Ribas et al., 2007; Caparroz et al., 2009; Chaves et al., 2014). Also, the parents of the specimen whose mitogenome is being described here were apprehended from the illegal trade and their origin is unknown. Therefore, in order to identify the possible origin of this specimen, we added its partial cytochrome oxidase I (COI) sequence into a matrix of 108 sequences of $506 \mathrm{bp}$ from individuals of the A. aestiva/A. ochrocephala complex with known geographic origin and available in GenBank (Eberhard et al., 2004; Ribas et al., 2007; Caparroz et al., 2009) (Table S3). The alignment was performed using ClustalW in MEGA6. The haplotype network was constructed by median joining using Network v 4.6 (Bandelt et al., 1999). We also used the webpage DNA Surveillance for species identification of Brazilian parrots (Baker et al., 2003; Chaves et al., 2014) to verify the species of our specimen based on their ND2 sequence. To ratify the other two approaches we aligned the COI gene sequences from 108 Amazona specimens (Table S3) using MUSCLE (Edgar, 2004). The resulting alignment contained 622 sites, 29 of which were variable and 17 informative. We estimated the coalescent history of the mitochondrial genomes of the Amazona aestiva based on this alignment using BEAST v1.8.4 (Drummond et al., 2012). We used a HKY+G nucleotide substitution model and the skyline plot model coalescent process (Drummond et al., 2005). We assumed a strict molecular clock, and calibrated the tree using a lineage-specific evolutionary rate for the Amazonas sp. (1.2510-8 substitutions per site per year), as inferred by Nabholz et al. (2016). We ran three MCMC chains for 20 million states, with a $10 \%$ burn-in. We inspected the results of all chains to observe for convergence in Tracer v1.6.

We also estimated Tajima's D neutrality test (Tajima's $\mathrm{D}=1.031)$, with $p$-value $(\mathrm{p}=0.80)$ calculated using 1000 simulations in Arlequin v3.5 (Excoffier and Lischer, 2010). The $p$-value was not statistically significant, with no evidence for selection, and the observed frequency of rare alleles was similar to the expected value.

\section{Results}

\section{Read mapping and features of the mitochondrial genome of Amazona aestiva}

A total of 42,268 reads of $A$. aestiva mapped onto the mitochondrial genome of $A$. barbadensis (Table 1$)$. The assembled mitogenome was obtained from 19,005 reads, with an average coverage of 183 -fold and a maximum coverage of 302-fold. The resulting $A$. aestiva mitogenome consisted of a circular molecule of 18,853 bp (GenBank accession number NC_033336, Figure 1). The Light-strand (L-strand) presented 5,692 adenines, 5,986 cytosines, 2,681 guanines and 4,494 thymines. We found 41 mitogenomic features, including protein coding genes, pseudogenes, tRNAs, rRNAs and two control regions (Table S2).

We found that the mitochondrial L-strand, defined as the A+C-rich strand (Munn, 1975; Taanman, 1999; Vinograd et al., 1963), encodes the majority of the genes in the A. aestiva mitogenome. We annotated in the L-strand 14 tRNAs, 2 rRNA, 12 protein coding genes, 2 pseudogenes 


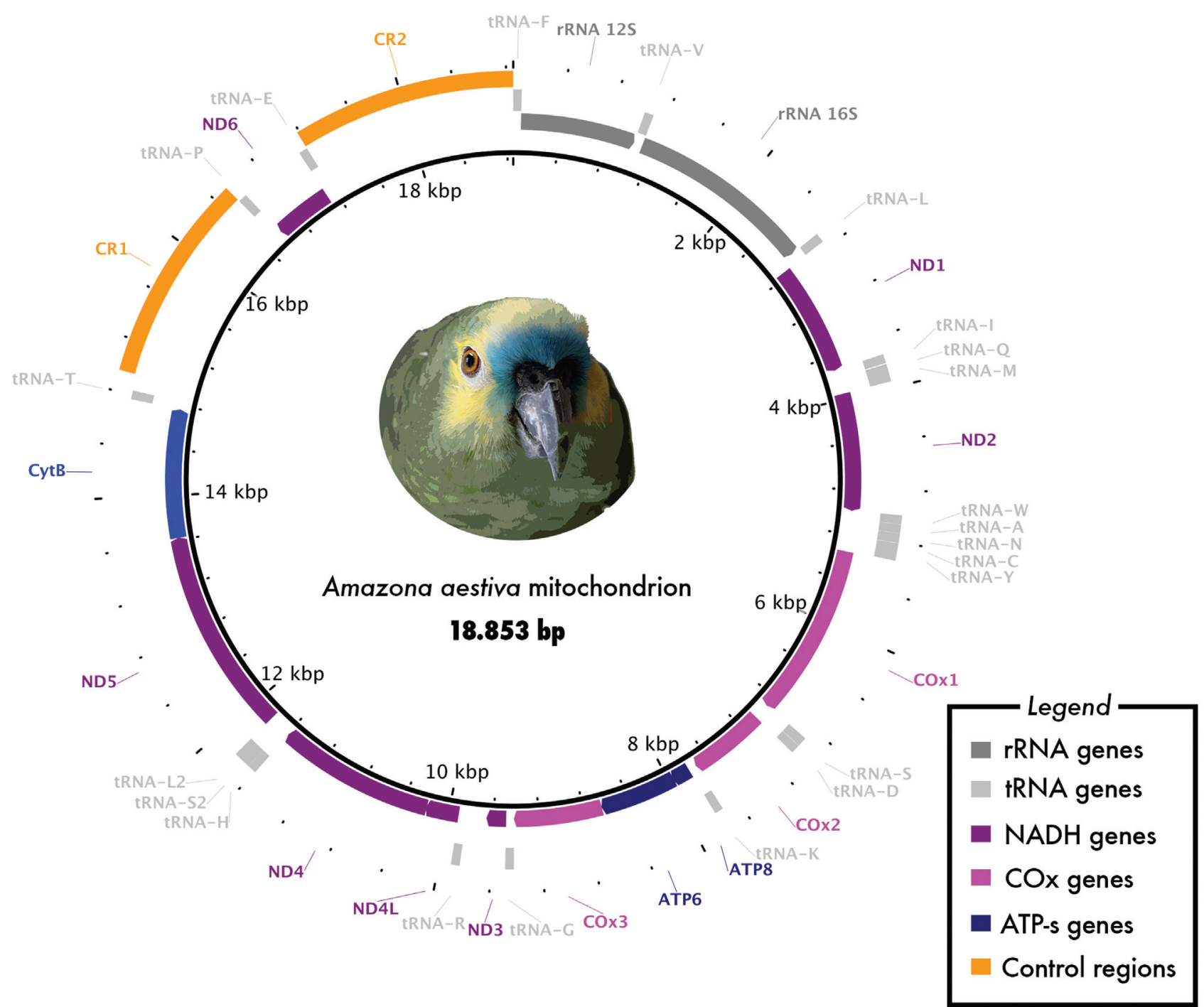

Figure 1 - Mitochondrial genome of Amazona aestiva. Major genomic features are shown. Colors follow the legend chart.

and 2 control regions (CRs). The Heavy-strand (H-strand) contained the remaining 8 tRNAs and 1 protein coding gene, that of NADH dehydrogenase subunit 6. Of the protein coding genes, 4 had stop codons after the polyadenylation site (Table S2, Figure 1), consistent with Ojala et al. (1981).

Consistent with the findings of Urantowka et al. (2013) and Eberhard and Wright (2016), we found that the mitogenome of $A$. aestiva follows the general Amazona mitochondrial gene order, confirming a CR duplication and pseudogenes for tRNA-Glu and ND6. Specifically, the gene order in this region was $\mathrm{CYTB} / \mathrm{tRNA}^{\mathrm{Thr}} / \mathrm{ND}^{\text {pseudo }}$ /

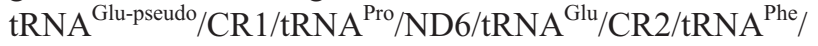

12s-rRNA. The two CRs of $A$. aestiva were shorter than the ones from $A$. barbadensis, and CR1 was shorter than CR2. The species difference in CR sizes is due to the different number of tandem repeats near the 3'-end of the CRs. The pseudogenes for ND6 and tRNA-Glu presented few differences in comparison with the $A$. barbadensis ones.
Description of control region domains and conserved sequence motifs

In vertebrates, CRs are normally divided into three domains (Figure 2). Domain I contains Extended Termination Associated Sequences (ETAS), domain II presents sequence blocks named from A to F, and domain III has conserved sequence blocks (CSB) numbered from 1 to 3 (Ruokonen and Kvist, 2002). In the $A$. aestiva mitogenome we observed that the consensus sequences of sequence boxes and CBSs are quite similar to each other, despite some differences (Table 3). In domain I we identified the poly-C tract (Table 2), with a central TA pair conserved in all CRs but with varying numbers of $\mathrm{Cs}$. We could define ETAS sequences 1 and 2 by alignment of the homologous sequences of $A$. aestiva with those of $A$. ochrocephala auropalliata, obtained from Eberhard and Wright (2016). Domain II had five conserved boxes: F, D, C, Bird similarity box (Bsb) and B. All consensus sequences from these 


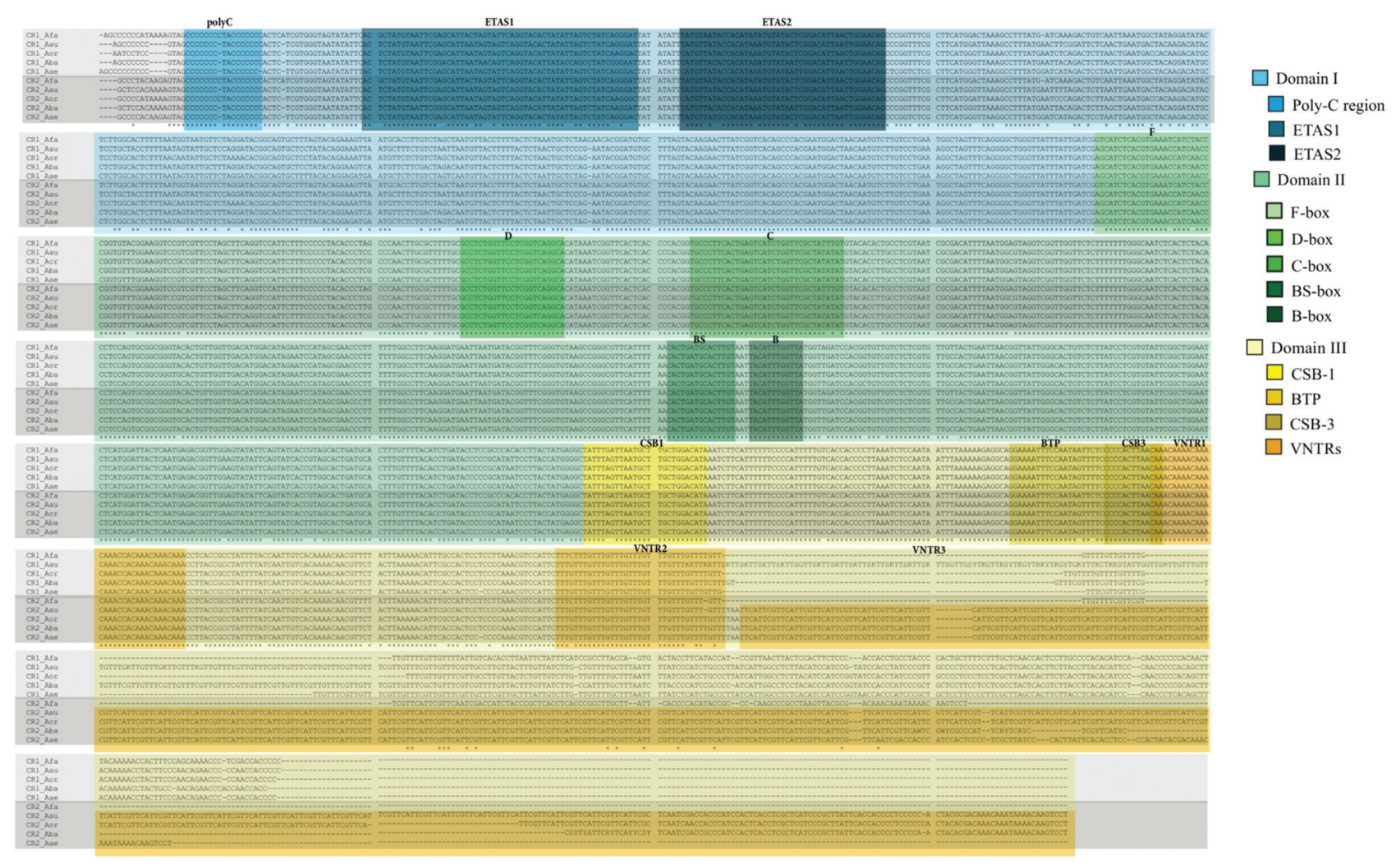

Figure 2 - Conserved motifs in the alignment of CRs of Amazona aestiva (Aae, KT361659), A. barbadensis (Aba, JX524615), A. farinosa (Afa, AF338821), A. ochrocephala oratrix (Aor, AF338820), and A. ochrocephala auropalliata (Aau AF338819).

boxes were conserved, with minor changes (Table 2, Figure 2). Domain III presented two out of three CSBs. CSB-1, which was used to define the border between domains II and III, was nearly identical in all Amazona species studied, with a shift from an internal $\mathrm{AG}$ to GA in the CRs of $A$. farinosa. As observed in other avian CRs, CSB-2 was absent (Desjardins and Morais, 1990). CSB-3 was identical among all CRs compared. We also identified a palindromic sequence that may be the bidirectional transcription promoter as verified by Eberhard and Wright (2016). Domain III also presented three different microsatellite repeats referred to as variable number tandem repeats (VNTRs). VNTR1 presented four CAAA repeats followed by a single internal repeat of CCA and three more CAAA repeats, and was conserved in all Amazona CRs compared. VNTR2 presented eight tetranucleotide microsatellite repeats of TTTG that were conserved in all species except in A. farinosa, whose first repeat was TTTC. VNTR3 was composed of several repetitions of an 8 nucleotide motif (TTCATTCG) that was absent in all CR1s but present in almost all CR2s, except for that of $A$. farinosa. Most species differences in CRs within the Amazona genus were in Domain III (similarity of $76 \%$ over 367 sites without gaps; 824 total sites) and were due to the VNTRs, which varied in length. Domain II was highly conserved (similarity of $93.9 \%$ over 609 sites without gaps; 610 total sites) and Domain I showed considerable variation (similarity of $78 \%$ over 431 sites without gaps; 436 total sites), though not as much as observed in Domain III. Within the same species, the two CR copies were almost identical in Domains I and II, but presented size and repeat differences in Domain III (Table 2 and Figure 2).

\section{Comparative mitogenomics of Amazon parrots}

Alignment of the complete mitogenomes of Amazon parrots (Amazona aestiva, A. barbadensis and $A$. ochrocephala) revealed greater differences between $A$. aestiva and $A$. barbadensis, with 523 mismatches, including indels. Most of these mismatches were in coding regions. In contrast, $A$. aestiva and A. ochrocephala presented 236 mismatches, mostly in the CR.

Comparative analyses of individual loci from the three species revealed that ND5 had the highest number of mismatches, most of them synonymous. It was possible to identify 3 indels at the $\mathrm{C}$-terminal of the A. barbadensis ND5. This includes a CT insertion that resulted in a frameshift leading to the substitution of a methionine codon for a leucine codon as well as a premature stop codon. However, since this insertion occurs within the 10 last predicted residues, it is possible that it does not cause major problems in protein folding or function (see also Tables S4, $\mathrm{S} 5$ and S6). Species differences in tRNAs and rRNAs were not common but were more frequent between $A$. aestiva and $A$. barbadensis. 
Table 2 - Number of single nucleotide polymorphisms when comparing the mitogenome of $A$. aestiva against the mitogenomes of $A$. barbadensis and $A$. ochrocephala.

\begin{tabular}{lcc}
\hline Mitogenome feature & $\begin{array}{c}\text { A. aestiva/ } \\
\text { A. barbadensis }\end{array}$ & $\begin{array}{c}\text { A. aestiva / } \\
\text { A. ochrocephala }\end{array}$ \\
\hline CDS* & 272 & 92 \\
rRNA & 10 & 3 \\
tRNA & 32 & 9 \\
Control Region & 203 & 130 \\
Transitions & 405 & 158 \\
Transversions & 27 & 9 \\
Indel* & 91 & 69 \\
Synonymous & 226 & 74 \\
Missense & 46 & 18 \\
Nonsense & 0 & 0 \\
Codon Position 1 & 49 & 14 \\
Codon Position 2 & 16 & 8 \\
Codon Position 3 & 207 & 70 \\
\hline
\end{tabular}

* $\mathrm{CDS}=$ coding sequence; Indel $=$ nucleotide insertion or deletion

\section{Psittaciformes phylogeny}

The phylogeny tree obtained (Figure 3 ) is in accordance with previously published phylogenies of Psittaciformes (Tavares et al., 2006; Schirtzinger et al., 2012). As expected, the outgroups (except for chicken, which was used to root the trees) were placed outside the Psittaciformes ingroup. Furthermore, the sister-taxa relationship between Psittaciformes and Passeriformes was recovered and is congruent with phylogenomic results (Jarvis et al., 2014). Some particular clades were recovered with high support inside the Psittaciformes clade. These were, first, the New Zealand parrots that clustered Strigops habroptilus and Nestor notabilis in a previously described basal clade of Psittaciformes (Tavares et al., 2006; Wright et al., 2008). A second well-supported clade contained various species of cockatoos from Australasia, consistent with its monophyly. The last higher clade recovered with high support contained all Amazona sampled.

The close relationship between the genera Cacatua and Calyptorhynchus, the genera Amazona (Eberhard et al., 2004; Ribas et al., 2007) and Ara (Schweizer et al., 2014) were recovered as previously described. Our results were also congruent with the recent taxonomic proposal for the genera Psittacara, Thectocercus, Eupsittula and Aratinga (Remsen Jr et al., 2013).

\section{Analysis of taxonomic status}

The specimen sequenced here presents a typical external morphology of Amazona aestiva aestiva (Figure S2). The haplotype network based on mitochondrial COI sequences separated this individual (H_1 in Figure 4) by a single mutation from individuals of $A$. aestiva from the Bra-

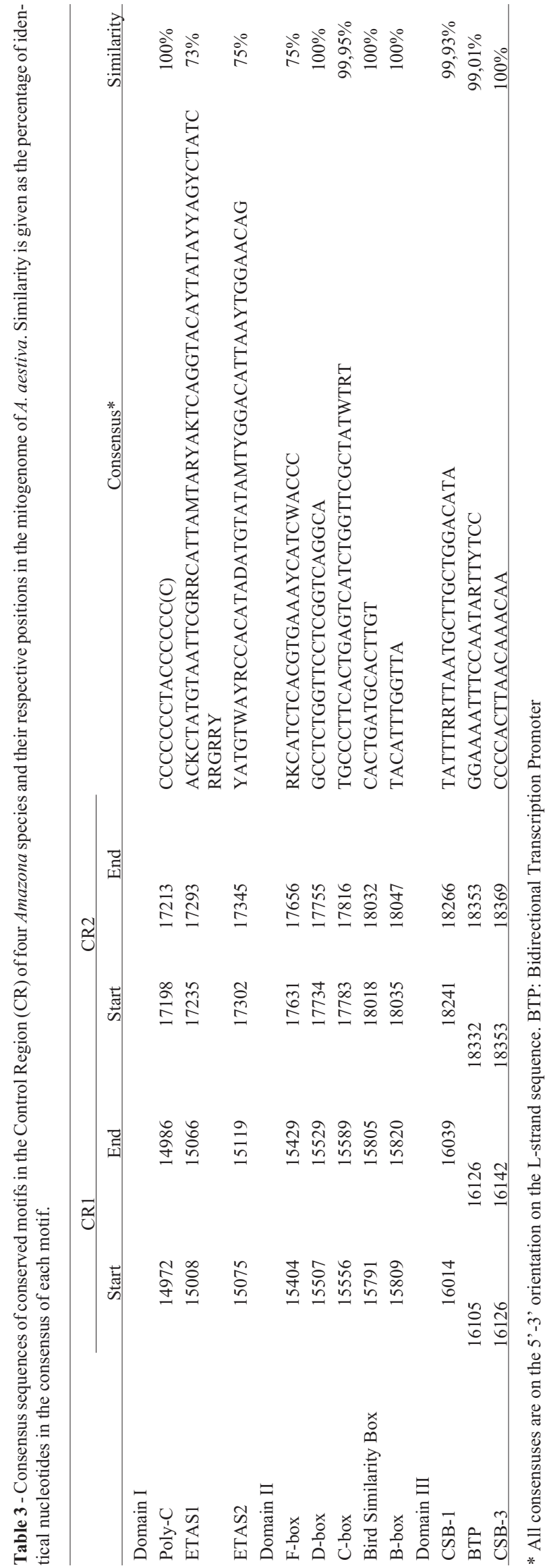




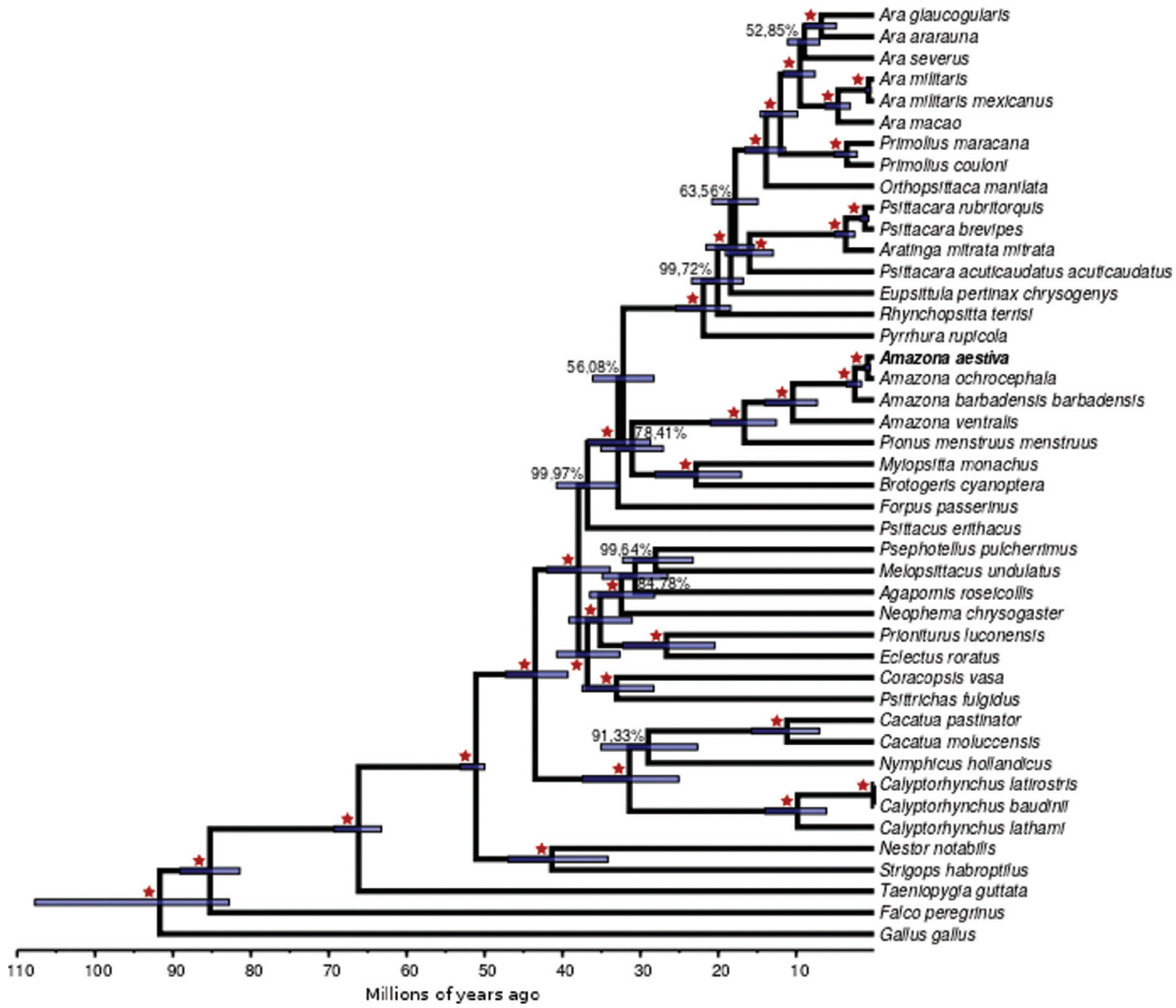

Figure 3 - Bayesian tree from all Psittaciformes with mitogenome available and three outgroups. Red stars mark branches with $100 \%$ support. Values lower than $100 \%$ are shown.

a.

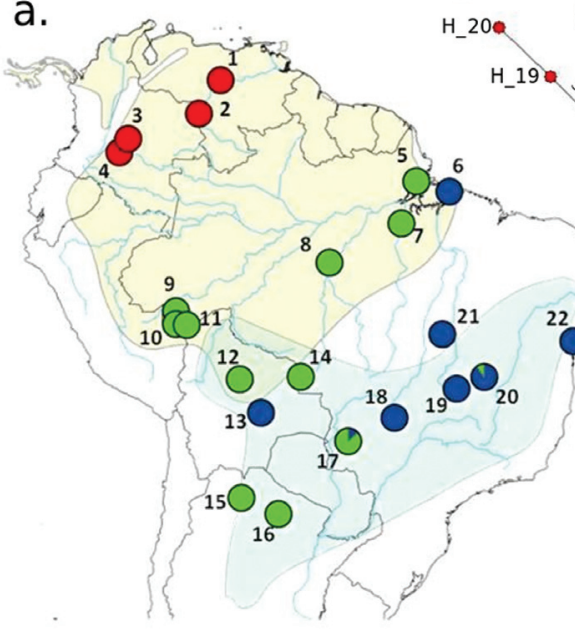

b.<smiles>[AlH2]</smiles>

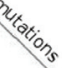

H_170 H_180 S H_22

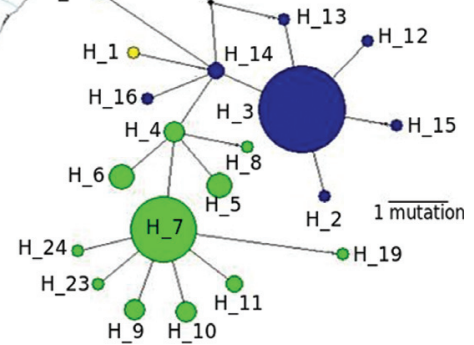

Figure 4 - Map and haplotype network. (a) Map showing the distribution of A. aestiva (light blue) and A. ochrocephala (yellow). Circles and numbers indicate the localities sampled (Table S3). Circle colors represent the frequency of observed lineages on the haplotype network for each locality. (b) Haplotype network with colors representing the three main lineages. Red - A. ochrocephala; Green - mixed population of A. ochrocephala and A. aestiva, with the majority being from the former; Blue - mixed population of $A$. aestiva and $A$. ochrocephala, with the majority being from the former. The COI sequence from the individual whose mitogenome was described (FVVF132) is in yellow. 
zilian state of Bahia, haplotype 14 (H_14, Figure 4). Unfortunately, the subspecies of the individuals with $\mathrm{H}_{-} 14$ is unknown (no information in GenBank records, nor in associated publications). The DNA Surveillance tree (Figure 5) placed this individual as closely related to the H9_clade1_SA, which presents sequences of captive $A$. aestiva specimens from the Brazilian states Bahia, Tocantins, Minas Gerais, Distrito Federal, and Goiás. The tree built from COI sequences using BEAST (Figure 6) placed this individual in a branch closely related to COIs from haplotype $\mathrm{H} \_14$ and $\mathrm{H} \_$16, which are also separated from H_14 by one mutation.

\section{Discussion}

We have assembled the complete mitogenome of $A$. aestiva by selecting mitochondrial reads from the whole genome sequencing reads. The majority of the protein coding genes and tRNAs were found to be encoded by the strand that presents the highest amount of $\mathrm{A}+\mathrm{C}$, the Lstrand (Lima and Prosdocimi, 2018). We recovered the two copies of the CR, which is known to be an AT-rich sequence implicated in the initiation and termination of replication and transcription (Shadel and Clayton, 1997; Gibson et al., 2005). In this region, a truncated $\mathrm{H}$-strand replication product (7S DNA) pairs with its complementary sequence on the L-strand and displaces the $\mathrm{H}$-strand forming a loop, known as displacement-loop or simply D-loop (Nicholls and Minczuk, 2014). Based on the chicken mitogenome (Schirtzinger et al., 2012), the ancestral location of the CR in the avian mitogenome is thought to be between the
tRNA-Pro and tRNA-Phe, which corresponds to the Amazon parrot CR2. Thus, the CR conventionally described as CR1 is likely a result of a duplication event that inserted this CR1 and its associated pseudogenes into the mitochondrial genome of an ancestral parrot population that gave rise to the genus Amazona. Based on the mitogenome of $A$. aestiva, we suggest that the 1,692 bases between the end of the tRNA-Thr and the start of the tRNA-Pro most likely correspond to the duplicated block. Differences in the length of two CRs in Amazon parrots are due to the presence of a variable number of tandem repeats on the 3 '-end (Figure 2).

The two mitochondrial CRs of the genus Amazona share an overall $84,5 \%$ identity, both within and across species, with high conservation of sequence motifs. This observation is consistent with the possibility that both CR copies may be functional and appear to be evolving in concert, as originally suggested by Eberhard et al. (2001). In chicken, the preferred start sites for mitochondrial DNA replication are regions flanking the CR (Reyes et al., 2005). If the same is true for Amazona and if both CR copies are functional, it is possible that the mitogenome of this genus may have a higher replication rate when compared to other mitochondria with single CRs. The presence of more efficient mitochondrial DNA replication could in principle increase the expression of components of the electron transport chain, which would concur with the high metabolic rates of parrots (Munshi-South and Wilkinson, 2010). Interestingly, preliminary genomic results by Wirthlin et al. (2018) point to selective pressure in superoxide dismu-

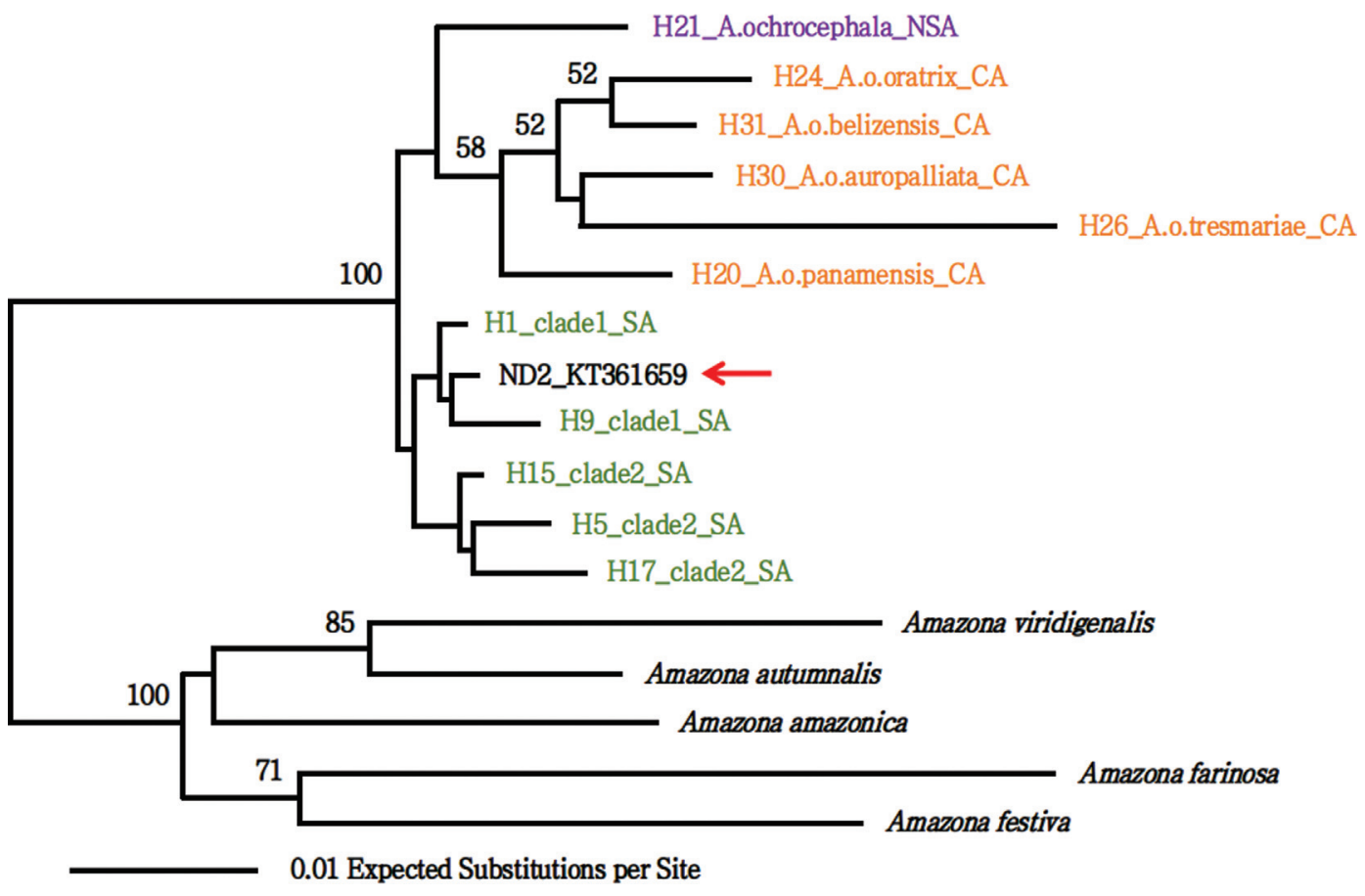

Figure 5 - Tree of the DNA Surveillance for species identification of Brazilian parrots. The ID in bold and marked with a red arrow corresponds to the individual analyzed here (FVVF132). Note that the closest related haplotype is H9_clade1_SA. 


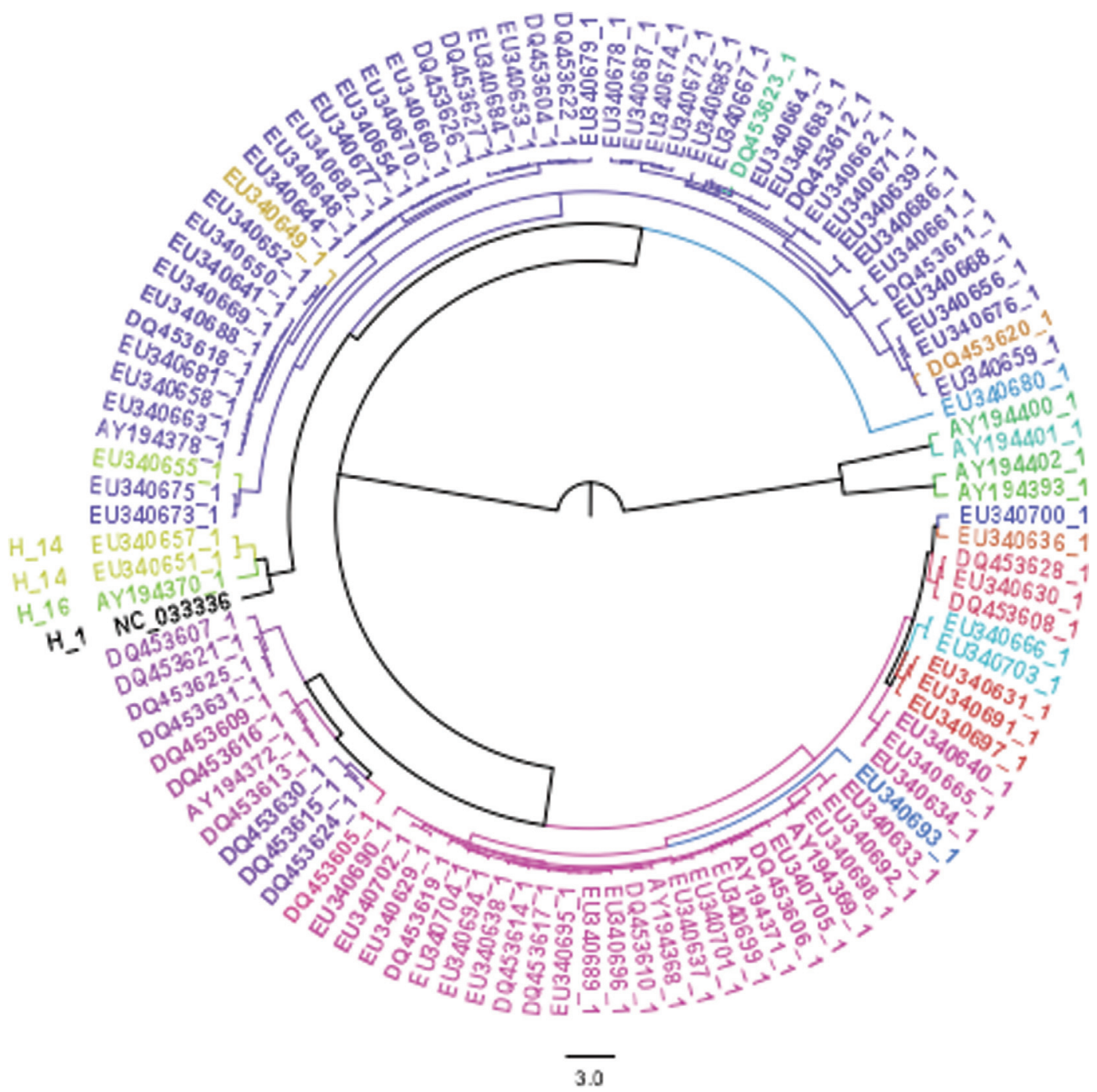

Figure 6 - Coalescent-based COI tree. The placement of the COI sequence from the individual described here (H_1, NC_033336) is consistent with our findings using the haplotype network and the DNA Surveillance identification of Brazilian parrots. We also marked the three closest sequences from haplotypes H_14 and H_16.

tase genes, suggesting the importance of antioxidant protection mechanisms in these birds.

An overall high level of sequence similarity was observed between complete Amazona mitogenomes. The paucity of polymorphisms in tRNA may indicate conservation of structure and, hence, function. Importantly, the majority of differences within the coding sequence are at the third codon position, leading to synonymous substitutions. The majority of first codon polymorphisms are also synonymous. The presence of an intergenic region between ND5 and CYTB in A. barbadensis was due to an insertion of CT creating a premature stop codon. This insertion seems to have occurred after this species diverged from $A$. aestiva and $A$. ochrocephala, since these two species share the same ND5 amino acid sequence, as well as the overlap of the 3'-end of ND5 and the 5'-end of CYTB.

The phylogeny reported here is congruent with a partitioned Bayesian analysis of 117 parrot species based on two mitochondrial genes, two nuclear introns and coded gaps (Schirtzinger et al., 2012). As expected, A. aestiva was shown to be a sister group of $A$. ochrocephala.

Analysis of the cytochrome oxidase I (COI) placed the bird, whose mitogenome was described here, close to H_14 from the state of Bahia in Brazil. H_14 contains the majority of COI's from $A$. aestiva mitogenomes. The H9 clade1_SA, which is the haplotype closest to our sequence by the DNA Surveillance classification (Figure 5), is part of clade 1, which according to Chaves et al. (2014), is related to the north-eastern group of Caparroz et al. (2009). The latter corresponds to the Brazil states Bahia, Tocantins, Minas Gerais, Distrito Federal, and Goiás. These placements were also confirmed by a phylogenetic tree using the same data. Thus, the specimen whose mitogenome we describe in the present study could be closely related to individuals from the state of Bahia. Also, H9_clade1_SA corresponds to captive A. aestiva aestiva 
specimens, which is consistent with our morphological subspecies classification.

In sum, the mitochondrial genome of the blue-fronted Amazon, A. aestiva, was described here for the first time and compared to other whole mitogenomes from the genus Amazona. Our data provide a new and high quality mitogenome of an Amazona species. Our analyses confirm the presence of conserved sequences and boxes in the duplicated control regions, providing support to previous studies on the organization of this mitogenomic feature. Our data also provide further support for the concerted evolution of these duplicated CRs. The phylogeny confirmed previous findings (e.g., sister relationship between Psittaciformes and Passeriformes, monophyly and basal position of Strigopoidae, monophyly of the Australasian cockatoos, monophyly of subfamily Arinae). Lastly, our data help clarify the haplotype placement of the specimen analyzed, confirming the subspecies classification based on morphological traits.

\section{Acknowledgments}

This work was sponsored by Brazilian funding agencies CNPq (SISBIOAVES project), FAPESPA, FAPEMIG, FAPESP (Biota 2013/50297-0), and FAPERJ (E-26/202.810/2015), as well as by NSF (DOB 1343578) and NASA. We gratefully acknowledge all authors from the $A$. aestiva genome paper, Drs. Morgan Wirthlin, Rafael Lucas Muniz Guedes, Nathalia P. Cavaleiro, Guilherme Loss de Morais, Anderson V. Chaves, Marcus de Melo Teixeira, Michael C. Schatz, Maria Sueli Felipe, and especially Erich Jarvis and Jason Howard from Duke University. Also, we would like to thank biologist Paulo Augusto Ribeiro Machado for providing access to the A. aestiva specimen (FVVF132) and Anderson Vieira Chaves for critically reading the manuscript.

\section{References}

Baker CS, Dalebout ML, Lavery S and Ross HA (2003) www.DNA-surveillance: Applied molecular taxonomy for species conservation and discovery. Trends Ecol Evol 18:271-272.

Bandelt HJ, Forster P and Röhl A (1999) Median-joining networks for inferring intraspecific phylogenies. Mol Biol Evol 16:37-48.

Bernt M, Donath A, Jühling F, Externbrink F, Florentz C, Fritzsch G, Pütz J, Middendorf M and Stadler PF (2013) MITOS: Improved de novo metazoan mitochondrial genome annotation. Mol Phylogenet Evol 69:313-319.

Boore JL (1999) Animal mitochondrial genomes. Nucleic Acids Res 27:1767-80.

Borsari A and Ottoni EB (2005) Preliminary observations of tool use in captive hyacinth macaws (Anodorhynchus hyacinthinus). Anim Cogn 8:48-52.

Brauth SE, Heaton JT, Shea SD, Durand SE and Hall WS (1997) Functional anatomy of forebrain vocal control pathways in the budgerigar (Melopsittacus undulatus). Ann N Y Acad Sci 807:368-385.

Caparroz R, Seixas GHF, Berkunsky I and Collevatti RG (2009) The role of demography and climatic events in shaping the phylogeography of Amazona aestiva (Psittaciformes, Aves) and definition of management units for conservation. Divers Distrib 15:459-468.

Chaves AV, Queiroz-Filho ROP, Silva FAA, Miyaki CY and Santos FR (2014) An online mtDNA tool for identification of Neotropical Psittacid species and taxonomic issues: A study case of the Amazona ochrocephala complex. Nat Resour 5:634-652.

Desjardins P and Morais R (1990) Sequence and gene organization of the chicken mitochondrial genome. J Mol Biol 212:599-634.

Drummond AJ, Rambaut A, Shapiro B and Pybus OG (2005) Bayesian coalescent inference of past population dynamics from molecular sequences. Mol Biol Evol 22:1185-1192.

Drummond AJ, Ho SYW, Phillips MJ and Rambaut A (2006) Relaxed phylogenetics and dating with confidence. PLoS Biology 4:e88.

Drummond AJ, Suchard MA, Xie D and Rambaut A (2012) Bayesian phylogenetics with BEAUti and the BEAST 1.7. Mol Biol Evol 29:1969-1973.

Eberhard JR and Wright TF (2016) Rearrangement and evolution of mitochondrial genomes in parrots. Mol Phylogenet Evol 94:34-46.

Eberhard JR, Wright TF and Bermingham E (2001) Duplication and concerted evolution of the mitochondrial control region in the parrot genus Amazona. Mol Biol Evol 18:1330-1342.

Eberhard JR, Bermingham E and Zink R (2004) Phylogeny and biogeography of the Amazona ochrocephala (Aves: Psittacidae) complex. Auk 121:318-332.

Edgar RC (2004) MUSCLE: Multiple sequence alignment with high accuracy and high throughput. Nucleic Acids Res 32:1792-1797.

Excoffier L and Lischer HEL (2010) Arlequin Suite Ver 3.5: A new series of programs to perform population genetics analyses under Linux and Windows. Mol Ecol Resourc 10:564-567.

Farabaugh SDR (1996) Acoustic communication in parrots: laboratory and field studies of budgerigars, Melopsittacus undulatus. In: Kroodsma DE and Miller EH (eds), Ecology and Evolution of Acoustic Communication in Birds. Cornell University Press, New York, pp 97-117.

Gernhard T (2008) The conditioned reconstructed process. J Theor Biol 253:769-778.

Gibson A, Gowri-Shankar V, Higgs PG and Rattray M (2005) A comprehensive analysis of mammalian mitochondrial genome base composition and improved phylogenetic methods. Mol Biol Evol 22:251-264.

Gouy M, Guindon S and Gascuel O (2010) SeaView Version 4: A multiplatform graphical user interface for sequence alignment and phylogenetic tree building. Mol Biol Evol 27:221-224.

Ingman M and Gyllensten U (2009) Vertebrate mitochondrial DNA. Rev Cell Biol Mol Med 2:57-134.

Jarvis ED, Mirarab S, Aberer AJ, Li B, Houde P, Li C, Ho SYW, Faircloth BC, Nabholz B, Howard JT, et al. (2014) Wholegenome analyses resolve early branches in the tree of life of modern birds. Science 346:1320-1331. 
Kurtz S, Phillippy A, Delcher AL, Smoot M, Shumway M, Antonescu C and Salzberg SL (2004) Versatile and open software for comparing large genomes. Genome Biol 5:R12.

Lanfear R, Frandsen PB, Wright AM, Senfeld T and Calcott B (2017) PartitionFinder 2: New methods for selecting partitioned models of evolution for molecular and morphological phylogenetic analyses. Mol Biol Evol 34:772-773.

Lima NCB and Prosdocimi F (2018) The heavy strand dilemma of vertebrate mitochondria on genome sequencing age: number of encoded genes or $\mathrm{G}+\mathrm{T}$ content? Mitochondrial DNA Part A 29:300-302.

Lowe TM and Eddy SR (1997) tRNAscan-SE: A program for improved detection of transfer RNA genes in genomic sequence. Nucleic Acids Res 25:955-964.

Milne I, Stephen G, Bayer M, Cock PJA, Pritchard L, Cardle L, Shaw PD and Marshall D (2013) Using Tablet for visual exploration of second-generation sequencing data. Brief Bioinform 14:193-202.

Mindell DP (1999) Avian molecular evolution and systematics. Condor 101:189.

Munn EA (1975) The structure of mitochondria. J Mol Cell Cardiol 7:713.

Munshi-South J, Wilkinson GS and Vega Rivera JH (2006) Diet influences life span in parrots (Psittaciformes). Auk 123:108-118.

Munshi-South J and Wilkinson GS (2010) Bats and birds: exceptional longevity despite high metabolic rates. Ageing Res Rev 9:12-19.

Nabholz B, Lanfear R and Fuchs J (2016) Body mass-corrected molecular rate for bird mitochondrial DNA. Mol Ecol $25: 4438-4449$.

Nicholls TJ and Minczuk M (2014) In D-loop: 40 years of mitochondrial 7S DNA. Exp Gerontol 56:175-181.

Ojala D, Montoya J and Attardi G (1981) tRNA punctuation model of RNA processing in human mitochondria. Nature 290:470-474.

Pepperberg IM (1990) Cognition in an African gray parrot (Psittacus erithacus): Further evidence for comprehension of categories and labels. J Comp Psychol 104:41-52.

Pepperberg IM (2002) In search of king Solomon's ring: Cognitive and communicative studies of grey parrots (Psittacus erithacus). Brain Behav Evol 59:54-67.

Pepperberg IM (2010) Vocal learning in grey parrots: A brief review of perception, production, and cross-species comparisons. Brain Lang 115:81-91.

Pepperberg IM and Funk MS (1990) Object permanence in four species of psittacine birds: An African Grey parrot (Psittacus erithacus), an Illiger mini macaw (Ara maracana), a parakeet (Melopsittacus undulatus), and a cockatiel (Nymphicus hollandicus). Anim Learn Behav 18:97-108.

Plummer TK and Striedter GF (2002) Brain lesions that impair vocal imitation in adult budgerigars. $\mathrm{J}$ Neurobiol 53:413-428.

Prum RO, Berv JS, Dornburg A, Field DJ, Townsend JP, Lemmon EM and Lemmon AR (2015) A comprehensive phylogeny of birds (Aves) using targeted next-generation DNA sequencing. Nature 526:569-567.
Remsen JV, Schirtzinger EE, Ferraroni A, Silveira LF and Wright TF (2013) DNA-sequence data require revision of the parrot genus Aratinga (Aves: Psittacidae). Zootaxa 3641:296-300.

Reyes A, Yang MY, Bowmaker M and Holt IJ (2005) Bidirectional replication initiates at sites throughout the mitochondrial genome of birds. J Biol Chem 280:3242-50.

Ribas CC, Tavares ES, Yoshihara C and Miyaki CY (2007) Phylogeny and biogeography of yellow-headed and bluefronted parrots (Amazona ochrocephala and Amazona aestiva) with special reference to the South American taxa. Ibis 149:564-574.

Ruokonen M and Kvist L (2002) Structure and evolution of the avian mitochondrial control region. Mol Phylogenet Evol 23:422-432.

Rutherford K, Parkhill J, Crook J, Horsnell T, Rice P, Rajandream MA and Barrell B (2000) Artemis: Sequence visualization and annotation. Bioinformatics 16:944-945.

Schirtzinger EE, Tavares ES, Gonzales LA, Eberhard JR, Miyaki CY, Sanchez JJ, Hernandez A, Müeller H, Graves GR, Fleischer RC, et al. (2012) Multiple independent origins of mitochondrial control region duplications in the order Psittaciformes. Mol Phylogenet Evol 64:342-356.

Schweizer M, Hertwig ST and Seehausen O (2014) Diversity versus disparity and the role of ecological opportunity in a continental bird radiation. J Biogeogr 41:1301-1312.

Shadel GS and Clayton DA (1997) Mitochondrial DNA maintenance in vertebrates. Annu Rev Biochem 66:409-435.

Taanman JW (1999) The mitochondrial genome: structure, transcription, translation and replication. Biochim Biophys Acta 1410:103-123.

Tavares ES, Baker AJ, Pereira SL and Miyaki CY (2006) Phylogenetic relationships and historical biogeography of neotropical parrots (Psittaciformes: Psittacidae: Arini) inferred from mitochondrial and nuclear DNA sequences. Syst Biol 55:454-470.

Urantowka AD, Hajduk K and Kosowska B (2013) Complete mitochondrial genome of endangered yellow-shouldered Amazon (Amazona barbadensis): Two control region copies in parrot species of the Amazona genus. Mitochondrial DNA 24:411-413.

Vinograd J, Morris J, Davidson N and Dove WF (1963) The buoyant behavior of viral and bacterial DNA in alkaline $\mathrm{CsCl}$. Proc Natl Acad Sci U S A 49:12-17.

Wright TF, Schirtzinger EE, Matsumoto T, Eberhard JR, Graves GR, Sanchez JJ, Capelli S, Müller H, Scharpegge J, Chambers GK, et al. (2008) A multilocus molecular phylogeny of the parrots (Psittaciformes): Support for a Gondwanan origin during the cretaceous. Mol Biol Evol 25:2141-2156.

Young AM, Hobson EA, Lackey LB and Wright TF (2012) Survival on the ark: Life-history trends in captive parrots. Anim Conserv 15:28-43.

Zimin AV, Marçais G, Puiu D, Roberts M, Salzberg SL and Yorke JA (2013) The MaSuRCA genome assembler. Bioinformatics 29:2669-2677.

\section{Internet Resources}

AND riche en, http://abcis.cbs.cnrs.fr/ABIM/w3bb/d_abim/riche-adn.html (accessed in 2015). 
The IUCN Red List of Threatened Species. (Version 2015-3), http://www.iucnredlist.org.

Tracer (version 1.6), http://www.beast2.org/tracer-2/.

\section{Supplementary material}

The following online material is available for this article:

Table S1 - Species, NCBI accession IDs, and references of the mitogenomes used.

Table S2 - Mitochondrial genome features of the blue-fronted parrot Amazona aestiva.

Table S3 - Sequences analyzed in the taxonomic study. Table S4 -Mitochondrial gene differences between species.
Table S5 - Polymorphisms found in the whole mitogenome alignment of Amazona aestiva and Amazona barbadensis. Table S6 - Polymorphisms found in the whole mitogenome alignment of Amazona aestiva and Amazona ochrocephala. Figure S1 - Graph of the sum of adenines and guanines in 45 nucleotide windows

Figure S2 - Photo of individual FVVF132 ("Moisés") whose mitogenome was analyzed.

Associate Editor: Louis Bernard Klaczko

License information: This is an open-access article distributed under the terms of the Creative Commons Attribution License (type CC-BY), which permits unrestricted use, distribution and reproduction in any medium, provided the original article is properly cited. 